\title{
An individual urinary proteome analysis in normal human beings to define the minimal sample number to represent the normal urinary proteome
}

Xuejiao Liu ${ }^{1 \dagger}$, Chen Shao ${ }^{2 \dagger}$, Lilong Wei ${ }^{3}$, Jindan Duan ${ }^{4}$, Shuzhen Wu ${ }^{4}$, Xuewang $\mathrm{Li}^{1}$, Mingxi Li ${ }^{1^{*}}$ and Wei Sun ${ }^{4^{*}}$

\begin{abstract}
Background: The urinary proteome has been widely used for biomarker discovery. A urinary proteome database from normal humans can provide a background for discovery proteomics and candidate proteins/peptides for targeted proteomics. Therefore, it is necessary to define the minimum number of individuals required for sampling to represent the normal urinary proteome.
\end{abstract}

Methods: In this study, inter-individual and inter-gender variations of urinary proteome were taken into consideration to achieve a representative database. An individual analysis was performed on overnight urine samples from 20 normal volunteers (10 males and 10 females) by 1DLC/MS/MS. To obtain a representative result of each sample, a replicate 1DLCMS/MS analysis was performed. The minimal sample number was estimated by statistical analysis.

Results: For qualitative analysis, less than $5 \%$ of new proteins/peptides were identified in a male/female normal group by adding a new sample when the sample number exceeded nine. In addition, in a normal group, the percentage of newly identified proteins/peptides was less than $5 \%$ upon adding a new sample when the sample number reached 10. Furthermore, a statistical analysis indicated that urinary proteomes from normal males and females showed different patterns. For quantitative analysis, the variation of protein abundance was defined by spectrum count and western blotting methods. And then the minimal sample number for quantitative proteomic analysis was identified.

Conclusions: For qualitative analysis, when considering the inter-individual and inter-gender variations, the minimum sample number is 10 and requires a balanced number of males and females in order to obtain a representative normal human urinary proteome. For quantitative analysis, the minimal sample number is much greater than that for qualitative analysis and depends on the experimental methods used for quantification.

Keywords: Urinary proteome, Minimal sample number

\footnotetext{
*Correspondence: mingxili@hotmail.com; sunwei1018@hotmail.com

${ }^{\dagger}$ Equal contributors

'Department of Nephrology, Peking Union Medical College Hospital, Chinese Academy of Medical Sciences, No. 1 Shuaifuyan, Wangfujing Street, Beijing, China

Full list of author information is available at the end of the article
} 


\section{Introduction}

Human urine is mainly composed of shed cells, debris, and secreted components from the urinary tract as well as blood components that have passed through glomerular filtration and renal tubule reabsorption. Therefore, urine contains useful information not only regarding the kidney and urinary tract, but also about more distant organs. Analysis of the urinary proteome could aid the discovery of biomarkers for both urogenital and systemic diseases. Moreover, compared to serum, human urine is relatively simple and easy to collect, which makes urinary proteome analysis an attractive approach in clinical proteomics research.

Because inherent and environmental factors may influence the components of the urinary proteome, the biological and technical variations are important issues for urinary proteome research. Many groups [1-6] have contributed data regarding this issue and found that (1) a considerable degree of variation can be found in intraday (collection from one volunteer at different daily time points), intra-individual (collection from one volunteer on different days), and inter-individual (collection from different volunteers) samples; (2) the variation of five intra-day samples (first morning, second morning, $24 \mathrm{~h}$, random, and water loading void) was similar; (3) the variation of intra-individual samples was less than that of inter-individual samples; and (4) technical variation was less than biological variation. Although great variations have been found in different urinary samples, a number of urinary proteins were demonstrated to be consistently present in urine samples collected at different time points and from different individuals [4]. Moreover, Nagaraj et al. used healthy volunteers to construct a common dataset of 500 urinary proteins [5]. Taken together, the findings to date indicate that the urinary proteome is relatively stable and a good source for disease biomarkers.

Since the first urinary proteome analysis was published in 2001 [7], many clinical urinary proteome differential analyses have been reported, including analyses of samples from urogenital diseases (kidney transplantation [8], diabetic nephropathy [9], obstructive nephropathy [10], bladder cancer [11], prostate cancer [12], and others) and non-urogenital diseases (hematopoietic stem cell transplantation [13], coronary artery disease [14], and others).

A urinary proteome database from normal human samples plays an important role in biomarker discovery. In the discovery stage, a database could be used as a control for a disease group. In the validation stage, the candidate proteins/peptides could be selected from the database for MS-based or immuno-based validation. Many groups have analyzed the normal human urinary proteome using various approaches and have identified more than 2500 urinary proteins to date [15]. Analyses of the normal urinary proteome have usually analyzed pooled or individual samples from several volunteers [15-17]. However, due to the variations in the urinary proteome, it is still unknown whether these data represent the true pattern of the normal urinary proteome. If the sample number was less than the minimal number required for a representative database in a group, then an analysis may only represent the pattern of selected individuals and not the entire group, which would be misleading for subsequent studies. Therefore, to obtain a representative urinary proteome, it is necessary to define the minimal urinary sample number needed. To the best of our knowledge, such an analysis has not been conducted to date.

In this study, inter-individual and inter-gender variations were taken into consideration to achieve a representative urinary proteome. An individual urinary proteome analysis of 10 male and 10 female normal overnight samples from healthy volunteers was used to define the minimal samples number required. Because the data-dependent acquisition mode in LC/MS/MS analysis is biased against low abundance proteins [18], replicate experimental strategies are often used to obtain a comprehensive analysis [18-20], and therefore this strategy was also adopted for this study. To determine how many runs are necessary to obtain a comprehensive result for one urine sample by $1 \mathrm{DLC} / \mathrm{MS} / \mathrm{MS}$, a pooled sample from ten male samples was analyzed with forty runs. Based on these calculations, 10 male and 10 female urinary samples were then analyzed by replicate 1DLC/ MS/MS. For qualitative analysis by intra-gender and inter-gender analysis, the minimal sample number for male, female, and normal groups was estimated. For quantitative analysis, the variation of protein abundance was defined by spectrum count and western blotting methods. And then the minimal sample number for quantitative proteomic analysis was estimated. The overall workflow is shown in Figure 1.

\section{Materials and methods \\ Apparatus}

An LTQ XL mass spectrometer was purchased from Thermo-Fisher (San Jose, CA). A 1200 nano-HPLC system was obtained from Agilent (Foster, CA). An ADVANCE CaptiveSpray source for Thermo and C18 reverse phase capillary column was purchased from Michrom Bioresources (Auburn, CA). The microwave oven used in this study was solid-state Whirlpool model VIP271 (Shanghai, China), and the maximum output power was $850 \mathrm{~W}$.

\section{Reagents}

Deionized water from a MilliQ RG ultrapure water system (Bedford, MA) was used at all times. HPLC grade 


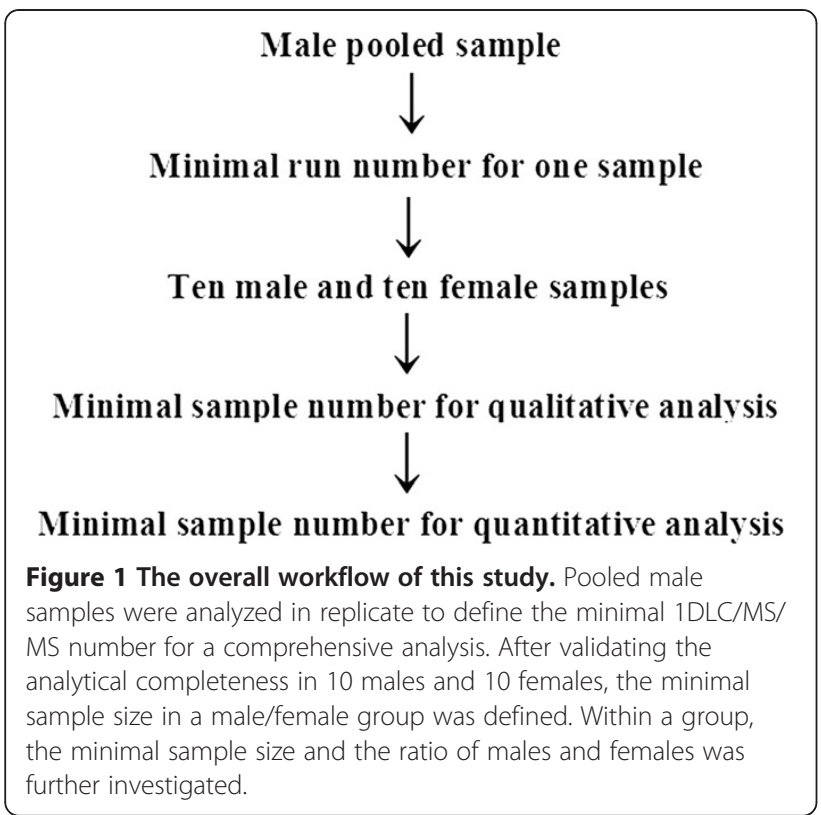

acetonitrile (ACN) and formic acid, ammonium bicarbonate, iodoacetamide, dithiothreitol (DTT), sequencing grade modified trypsin, and protease inhibitor phenylmethylsulfonyl fluoride (PMSF) were purchased from Sigma-Aldrich (St. Louis, MO).

\section{Urine collection}

Overnight urine samples were collected from twenty consenting individuals, including 10 males and 10 females (average age 28 and $31 \mathrm{y}$, respectively). The donors had no acute or chronic illnesses and were not taking any prescription or over-the-counter medications. No female was menstruating at the time of urine collection. Each specimen was collected in $250 \mathrm{~mL}$ conical tubes. The samples were immediately acidified to $\mathrm{pH} 2.7$ with hydrochloric acid and then cooled to $4{ }^{\circ} \mathrm{C}$ to prevent bacterial growth and proteolysis.

\section{Acetone precipitation}

All procedures were performed at $4{ }^{\circ} \mathrm{C}$. Urine samples were centrifuged at $5,000 \times \mathrm{g}$ for $30 \mathrm{~min}$ and the pellets were removed. The supernatants were then precipitated with $75 \% \mathrm{v} / \mathrm{v}$ acetone for $16 \mathrm{~h}$ followed by centrifugation at $12,000 \mathrm{x}$ g for $30 \mathrm{~min}$. The pellets were resuspended in lysis buffer (7 M urea, $2 \mathrm{M}$ thiourea, $50 \mathrm{mM}$ Tris, and $50 \mathrm{mM}$ dithioerythritol) and subjected to protein quantitation by the Bradford method. One pooled male sample was mixed using ten male samples with an "equal amounts of protein" criterion.

\section{Protein digestion}

Each sample was digested with trypsin as previously described [21]. Briefly, each sample was reduced with
DTT by heating at $100{ }^{\circ} \mathrm{C}$ for $5 \mathrm{~min}$ and then alkykated with iodoacetamide at room temperature in the dark for $45 \mathrm{~min}$. The samples were then digested with trypsin (1:50) for 1 min under microwave irradiation at $850 \mathrm{~W}$ using the following method: samples were placed into $1.5 \mathrm{~mL}$ polypropylene vials and a container with 1,000 $\mathrm{mL}$ of water was placed beside the sample vials to absorb the extra microwave energy. The microwave oven was turned on for $1 \mathrm{~min}$. After microwave irradiation, the vials were removed from the microwave oven and lyophilized to near dryness.

\section{DLC/MS/MS}

All lyophilized samples were redissolved in $0.1 \%$ formic acid (buffer A) at a concentration of $5 \mathrm{mg} / \mathrm{mL}$ before MS analysis. All peptide mixtures were analyzed on a reverse phase C18 capillary LC column from Michrom Bioresources $(100 \mu \mathrm{m} \times 150 \mu \mathrm{mm}, 3 \mu \mathrm{m}, 0.5 \mu \mathrm{L} / \mathrm{min})$. The elution gradient was $5-30 \%$ buffer B $(0.1 \%$ formic acid, 99.9\% ACN; flow rate, $0.5 \mu \mathrm{L} / \mathrm{min}$ ) for $100 \mathrm{~min}$. Eluted peptides were analyzed by an LTQ XL electrospray ion trap mass spectrometer. Ions were detected in a survey scan from 400 to $2000 \mathrm{amu}$ followed by 10 data-dependent MS/MS scans $(1 \mu$ scan each, isolation width $2 \mathrm{amu}, 35 \%$ normalized collision energy, dynamic exclusion for $90 \mathrm{~s}$ ) in a completely automated fashion.

\section{Western blot analysis}

Western blots were performed for three proteins: alpha 1 antitrypsin, ceruloplasmin, and beta-2-microglobulin to confirm the variation in urine. For each protein, 16 normal human overnight urine samples (10 females and 6 males) were used. A urine sample from a stage IV diabetic nephropathy patient was used as a control. Thirty micrograms urine protein from each urine sample after acetone precipitation was separated on a $4-12 \%$ NuPAGE gel (Invitrogen). Proteins were then transferred to polyvinylidene difluoride (PVDF) membranes (Millipore, USA). The membranes were blocked for $1 \mathrm{~h}$ at room temperature in Tris-buffered saline (TBS) with 5\% skim milk, and then incubated overnight at $4{ }^{\circ} \mathrm{C}$ in a $1 \%$ milk solution containing mouse monoclonal anti-alpha 1 antitrypsin (SERPINA1) antibody (1:1000, ab9400, Abcam), mouse monoclonal anti-ceruloplasmin antibody (1:1000, ab51083, Abcam), and rabbit monoclonal antibeta-2-microglobulin antibody (1:1000, ab15976, Abcam), respectively. The membranes were washed three times for $5 \mathrm{~min}$ with TBST (Tris-buffered saline with $0.05 \%$ Tween-20), and then incubated with horseradish peroxidase-labeled goat anti-mouse or mouse anti-rabbit IgG secondary antibodies (diluted 1:5000, Abcam) at room temperature for $3 \mathrm{~h}$. After washing three times for 5 min each in TBST, the membrane was visualized with an ECL detection kit (Millipore, Bedford, 
MA, USA) using a chemiluminescence imaging system (Millipore). Quantification of protein bands was performed for each sample by determining the relative optical density (ImageJ; National Institutes of Health, Bethesda, MD).

\section{Data processing}

MS/MS spectra were extracted from raw files requiring a minimum of 50 signals with an intensity of at least $1 \mathrm{x}$ $10^{5}$ U. Extracted MS/MS spectra were automatically assigned to the best-matching peptide sequences using the SEQUEST algorithm [22] and SEQUEST Browser software package Bioworks 3.3.1 SP1. SEQUEST searches were performed on a PC against an IPI human protein database (v3.70, released on 6th September, 2010) [23] containing 87,069 protein sequences downloaded as FASTA-formatted sequences from the Website of the European Bioinformatics Institute (http://www. ebi.ac.uk/IPI/). To increase search speed, the protein database was preprocessed to create a binary database containing all possible tryptic peptides. A static modification of $+57 \mathrm{Da}$ on cysteine residues was used. The peptide mass search tolerance was set to $1.4 \mathrm{Da}$. Because the number of methionine is relatively few in the database and adding a variable modification maybe significantly increase random match, especially for low resolution instrument (such as LTQ XL used in the work), therefore we did not use the variable modification of methionine oxidation to achieve more accurate database searching results.

Stringent SEQUEST filter criteria were used and included the following: (1) DeltaCn score of at least 0.2; (2) Rsp of 1; and (3) the XCorr cutoff was adjusted to maintain the average false positive rate of all datasets at approximately $1 \%$, leading to the following thresholds: 1.8, 2.8, and 3.3 for single, double, and triple-charged peptides, respectively.

To reduce redundancy in protein identification, peptides identified from tandem spectra were reassigned to proteins using the following procedure: (1) all peptides identified by MS/MS spectra were searched against protein databases to define whether each peptide was unique or shared (i.e., whether the peptide appeared in a single protein or in multiple proteins); (2) a protein identified by unique peptide(s) was marked as a single protein, and any peptide shared with this protein was also assigned to the protein; (3) the remaining shared peptides were redistributed to group proteins using the Occam's razor constraint [24], which states that the least number of proteins yields the most peptide sequences; and (4) in a group of proteins identified by the same shared peptides, the longest protein and/or the protein annotated by the Swiss-Prot or Trembl databases was chosen to represent the group.

\section{Statistical analysis}

To estimate the number of newly identified proteins along with the increased run/sample numbers, computer simulations were conducted by randomizing the order of each run/sample to be added to the existing run/sample pool. Means and standard deviations of the newly identified rates were calculated based on 5000 simulations to plot all of the saturation curves presented in this study. To further study the intra- and inter-gender variation, the overlap rate of identified proteins was calculated for each pair of samples. Hierarchical clustering analysis was also applied to this qualitative data. The distance between two samples was represented by one minus the overlap rate and the Ward's minimum variance method was used as the clustering method. All of the statistical analyses were performed using the $\mathrm{R}$ program.

\section{Quantitative analysis and sample size calculation}

Spectrum counts of proteins were calculated for the quantitative analysis. Since the LTQ XL mass spectrometer used in this study is of relative low sensitivity, only proteins that were reproducibly identified in at least $80 \%$ of the total $291 \mathrm{MS} / \mathrm{MS}$ runs were included in this study. To determine the minimal sample size needed to detect protein expression differences with a given level of statistical significance, the pwr.t.test function in the pwr package of the $\mathrm{R}$ program was used for power calculation of the two-sample $t$ test [25].

\section{Results}

\section{Overall identification of $\mathbf{2 1}$ samples}

To achieve a comprehensive analysis of the urinary proteome by $1 \mathrm{DLC} / \mathrm{MS} / \mathrm{MS}$, a replicate analysis was performed for 21 samples (one pooled male sample, 10 male samples, and 10 female samples). A total of 40 runs were performed for the pooled male sample, and an average of 12.5 runs were performed for each male/female sample, yielding a total of 291 runs for this study. To obtain high confidence results, a reverse databasesearching method was used to evaluate random matches. All raw data were searched against the reverse database to estimate the false-positive rate (the false-positive rate $=2 \mathrm{x}$ [spectrum count in reverse database] $/[$ spectrum count in reverse database + spectrum count in forward database] x 100 [\%]) [26]. The average false-positive rate of 291 results was approximately $1 \%$ based on a stringent SEQUEST criterion (Additional file 1).

In all, a total of 867 proteins, 2,804 peptides, and 152,449 spectra were identified from 21 samples, and an average of 219 proteins, 520 peptides, 7,259 spectra were found in each sample (Table 1, detailed information in Additional files 123 4). The inter-run overlap rates for protein and peptide identification were $74.12 \%$ and $71.23 \%$, respectively, indicating a good reproducibility of 
Table 1 The identification and inter-run overlap rates for $\mathbf{2 1}$ urinary samples

\begin{tabular}{|c|c|c|c|c|c|c|c|c|c|}
\hline & \multicolumn{3}{|c|}{ Protein } & \multicolumn{3}{|c|}{ Peptide } & \multicolumn{2}{|c|}{ Spectrum } & \multirow[t]{2}{*}{ Runs } \\
\hline & Mean \pm SD & Total & Overlap rate (\%) & Mean \pm SD & Total & Overlap rate (\%) & Mean \pm SD & Total & \\
\hline Pooled male sample & $86 \pm 6$ & 232 & $76.14 \pm 3.49$ & $167 \pm 11$ & 457 & $72.8 \pm 2.75$ & $412 \pm 39$ & 16,524 & 40 \\
\hline Male1 & $94 \pm 6$ & 188 & $77.17 \pm 3.04$ & $268 \pm 10$ & 481 & $79.09 \pm 1.99$ & $921 \pm 66$ & 11,054 & 12 \\
\hline Male2 & $122 \pm 4$ & 201 & $82.31 \pm 2.8$ & $324 \pm 8$ & 556 & $79.59 \pm 3.07$ & $863 \pm 26$ & 10,366 & 12 \\
\hline Male3 & $120 \pm 9$ & 225 & $79.62 \pm 2.73$ & $248 \pm 23$ & 494 & $76.02 \pm 3.63$ & $785 \pm 56$ & 10,213 & 13 \\
\hline Male4 & $150 \pm 9$ & 285 & $77.89 \pm 3.43$ & $395 \pm 20$ & 803 & $74.35 \pm 4.58$ & $985 \pm 55$ & 13,801 & 14 \\
\hline Male5 & $73 \pm 5$ & 129 & $79.36 \pm 3.12$ & $196 \pm 10$ & 374 & $75.72 \pm 2.83$ & $551 \pm 28$ & 5519 & 10 \\
\hline Male6 & $75 \pm 3$ & 132 & $78.17 \pm 2.64$ & $203 \pm 10$ & 363 & $76.89 \pm 1.91$ & $785 \pm 40$ & 7072 & 9 \\
\hline Male7 & $82 \pm 5$ & 160 & $75.21 \pm 3.3$ & $162 \pm 11$ & 329 & $71.9 \pm 2.24$ & $392 \pm 25$ & 3920 & 10 \\
\hline Male8 & $82 \pm 4$ & 162 & $74.34 \pm 3.2$ & $164 \pm 8$ & 336 & $71.09 \pm 2.56$ & $366 \pm 26$ & 3294 & 9 \\
\hline Male9 & $99 \pm 3$ & 197 & $76.36 \pm 2.59$ & $212 \pm 8$ & 438 & $73.15 \pm 2.54$ & $513 \pm 24$ & 5647 & 11 \\
\hline Male10 & $83 \pm 5$ & 167 & $77.23 \pm 2.38$ & $192 \pm 8$ & 397 & $73.82 \pm 1.78$ & $417 \pm 14$ & 5013 & 12 \\
\hline Female1 & $101 \pm 4$ & 243 & $69.83 \pm 2.85$ & $237 \pm 10$ & 558 & $72.21 \pm 1.78$ & $529 \pm 22$ & 7941 & 15 \\
\hline Female2 & $97 \pm 5$ & 212 & $69.6 \pm 3.19$ & $245 \pm 10$ & 565 & $67.85 \pm 2.52$ & $446 \pm 23$ & 4908 & 11 \\
\hline Female3 & $106 \pm 8$ & 226 & $68.99 \pm 2.91$ & $227 \pm 11$ & 550 & $61.68 \pm 2.19$ & $343 \pm 19$ & 3439 & 10 \\
\hline Female4 & $104 \pm 4$ & 217 & $72.42 \pm 2.61$ & $197 \pm 9$ & 479 & $67.75 \pm 1.77$ & $358 \pm 14$ & 3942 & 11 \\
\hline Female5 & $103 \pm 9$ & 254 & $67.72 \pm 4.07$ & $218 \pm 19$ & 588 & $63.89 \pm 4.33$ & $415 \pm 37$ & 6235 & 15 \\
\hline Female6 & $79 \pm 4$ & 180 & $75.13 \pm 2.74$ & $152 \pm 8$ & 363 & $70.85 \pm 2.51$ & $333 \pm 14$ & 5336 & 16 \\
\hline Female7 & $141 \pm 7$ & 357 & $70.55 \pm 2.36$ & $336 \pm 16$ & 867 & $69.56 \pm 1.71$ & $581 \pm 31$ & 9310 & 16 \\
\hline Female8 & $119 \pm 7$ & 297 & $68.27 \pm 2.84$ & $267 \pm 15$ & 723 & $64.17 \pm 2.38$ & $443 \pm 23$ & 6657 & 15 \\
\hline Female9 & $103 \pm 6$ & 238 & $70.37 \pm 3.3$ & $198 \pm 17$ & 506 & $66.88 \pm 3.82$ & $350 \pm 31$ & 4912 & 14 \\
\hline Female10 & $120 \pm 5$ & 296 & $69.97 \pm 3.75$ & $264 \pm 13$ & 701 & $66.67 \pm 3.98$ & $459 \pm 22$ & 7346 & 16 \\
\hline Total & & 867 & & & 2,804 & & & 152,449 & 291 \\
\hline
\end{tabular}

the 1DLC/MS/MS analysis. In this study, a replicate 1DLC/MS/MS strategy was used to improve detection sensitivity and to balance the high-abundance protein identification bias of the data-dependent acquisition mode. Compared with a single run, more than 2-fold protein/peptides were identified with the replicate strategy in 21 samples, providing more information on the samples for further analysis (Table 1).

\section{The minimal 1DLC/MS/MS number for a comprehensive analysis of one urine sample}

To estimate the minimal 1DLC/MS/MS number of runs required for a comprehensive analysis using the present instrumentation and protein database, replicate 1DLC/ MS/MS runs were statistically analyzed to determine at which point additional experiments produced fewer newly identified proteins. A total of 40 runs were performed in a pooled male sample and identified a total of 232 proteins and 457 peptides, with an average inter-run overlap rate of $76.14 \%$ (Table 1).

To estimate the percentage of newly identified proteins by increasing replicate run number, a saturation curve was plotted by computer simulation as described in the Method section. Figure 2 showed that approximately $76 \%$ of identified proteins in any run could be confirmed by any second run and $24 \%$ of proteins remained unconfirmed. Adding a third run decreased the newly identified protein percentage to $12 \%$. After six runs, this number decreased to $4.9 \%$, and after 25 runs,

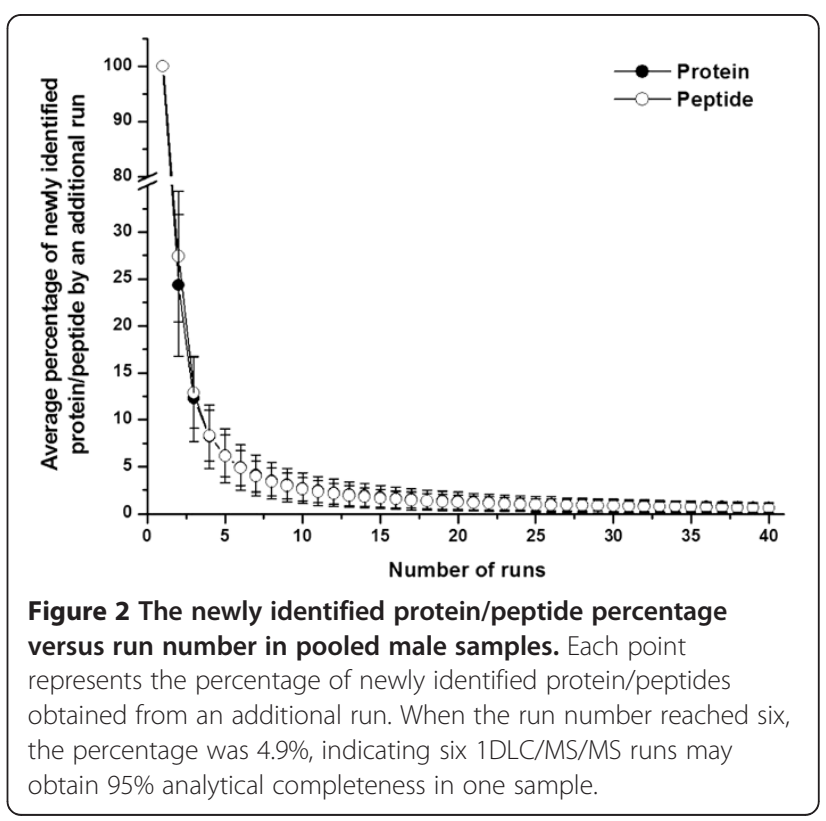


less than $1 \%$ of new proteins could be identified. Although 25 1DLC/MS/MS analyses provided more information than six analyses (208 proteins vs. 144 proteins, respectively), approximately 3 -fold more experiments had to be performed, which is not suitable for analyzing larger numbers of samples. Therefore, six 1DLC/MS/MS analyses were empirically chosen as the point for the subsequent individual urinary proteome analyses. The newly identified peptide percentage with the increase in the number of runs was also plotted and showed almost the same trend as that of proteins (Figure 2).

\section{The analytical completeness of 20 urine samples}

To define the minimal sample number for a group urinary proteome analysis, it is necessary to perform a comprehensive analysis of each sample. The term "analytical completeness" was used to describe the completeness of a urinary proteome obtained by LC/MS/MS analysis for one sample. The percentage of analytical completeness represented the percentage of newly identified protein/ peptides gained by an additional run.

Overnight urine samples from 10 males and 10 females were analyzed by replicate 1DLC/MS/MS and each sample was subjected to at least nine 1DLC/MS/ MS analyses. At a $1 \%$ false positive rate, a total of 836 proteins and 2,396 peptides (an average of 213 proteins and 522 peptides in each sample) were identified in these 20 samples, and the average inter-run overlap rate was $74.03 \%$ (Table 1 ).

To validate the analytical completeness of the 20 samples, the newly identified protein/peptide percentage was calculated as a function of run number. The results from the 20 samples (Additional file 5) showed that when the run number exceeded six, the newly identified protein/ peptide percentage decreased to less than $5 \%$, consistent with the male pooled sample, and at 12 and 15 1DLC/ MS/MS runs, the final newly identified protein/peptide percentage was approximately $2 \%$. These results indicate that by using replicate $1 \mathrm{DLC} / \mathrm{MS} / \mathrm{MS}$ analyses (9-16 runs), more than 95\% analytical completeness was achieved for the 20 samples.

\section{The minimal sample number for a male/female group by qualitative analysis}

Before estimating the minimal sample number for a group, the sample number for a sub-group was defined, which in this case was a male/female group. The intragender individual variation was defined by protein/peptide overlap rate analysis between the 20 samples. The average intra-gender protein/peptide overlap rates from 10 male and 10 female samples was 58.62\% and 50.71\%, respectively, which were significantly lower than that of inter-run rates (Figure 3). These results showed that there was intra-gender individual variation in the urinary

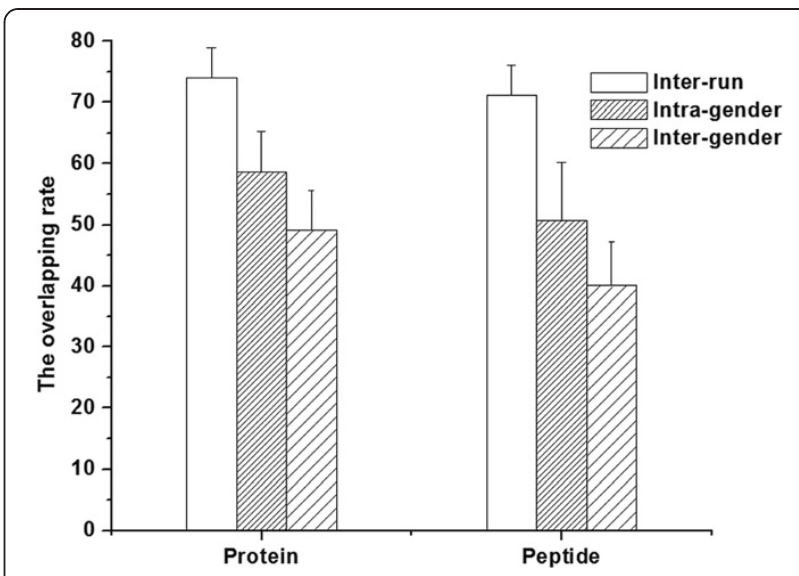

Figure 3 The comparison of average protein/peptide overlap rate from intra-run, intra-gender, and inter-gender analyses. The difference in the overlap rates of intra-run, intra-gender, and inter-gender analyses indicated there was intra- and inter-gender individual variation in the urinary proteome.

proteome, which were consistent with previous reports $[15,16]$. The intra-gender individual variation indicated that for a male/female group, multiple samples should be included in order to obtain a comprehensive urinary proteome analysis.

The 10 male or 10 female 1DLC/MS/MS results were then used to define the minimal sample number for a male or female group, respectively. The term "analytical completeness" was also used to describe the completeness of a group urinary proteome by multiple sample statistical analysis. The newly identified protein/peptide percentage was calculated as a function of sample size. The saturation curves for male and female samples were plotted by computer simulation. When the sample number was six, the newly identified protein/peptide percentage was less than $10 \%$, and when the sample number reached nine, the percentage decreased to less than $4 \%$. The results for males and females showed the same trend (Figure 4). These data indicated that 6/9 samples may achieve approximately $90 / 95 \%$ analytical completeness, respectively, of a male/female group urinary proteome.

\section{The minimal sample number for a group by qualitative analysis}

The inter-gender individual variations were also defined by a protein/peptide overlap rate analysis between all of the 20 samples. The overlap rate of inter-gender samples (49.12 and $40.02 \%$ for protein and peptide, respectively) was significantly lower than that of intra-gender samples (Figure 3), indicating that there was inter-gender individual variation in the urinary proteome. Moreover, a previous study [27] reported that gender-specific proteins could be found in male samples, suggesting that when 


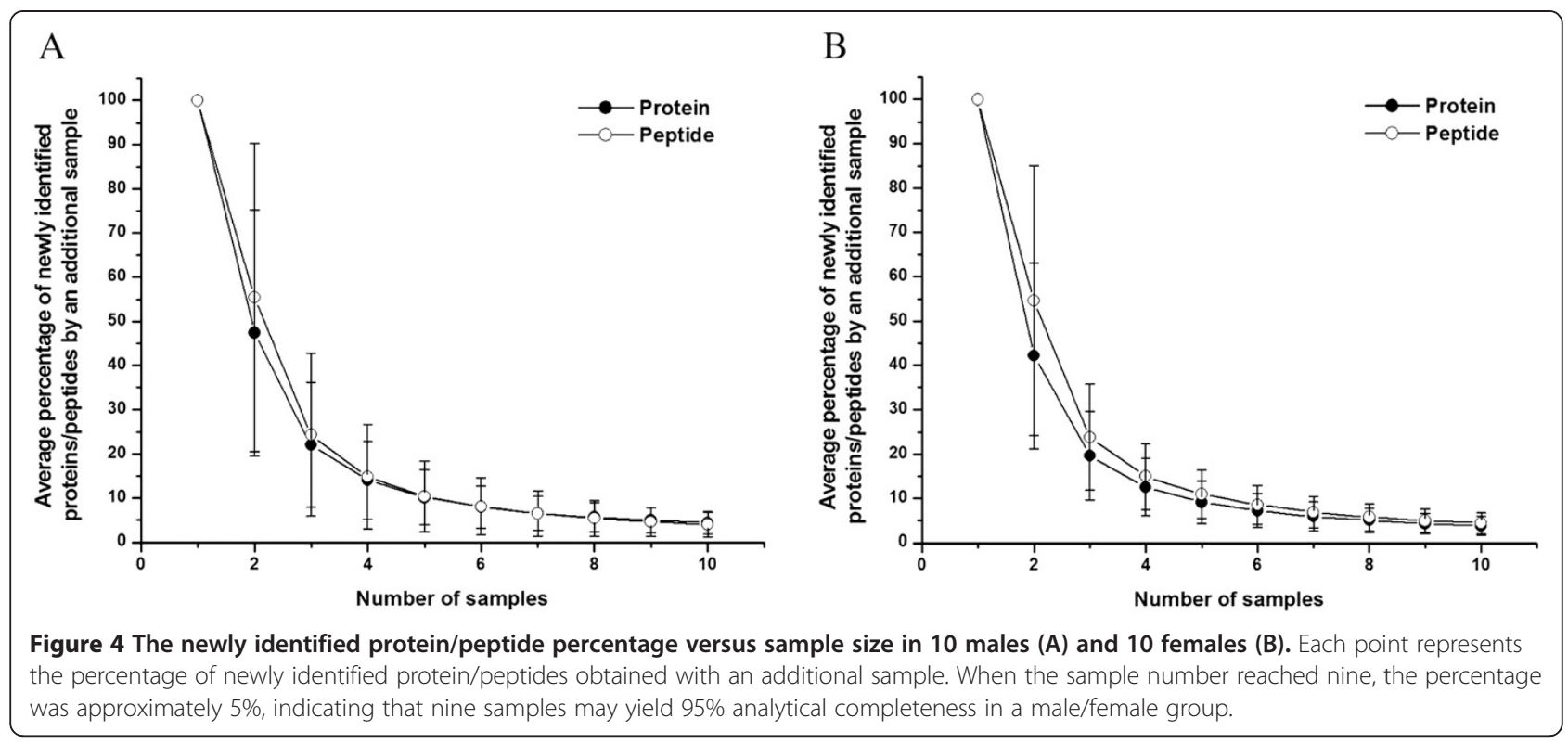

the minimal sample number for a group is determined by inter-gender sample analysis, the selected samples should include at least one male/female sample.

Figure 5 showed that when the sample number was six, the newly identified protein/peptide percentage was less than $10 \%$, and when the sample number reached 10 , the percentage decreased to less than $5 \%$. In addition, when the sample number was increased to 20 , the newly identified protein/peptide percentage was approximately $2 \%$. The above results showed that $6 / 10 \mathrm{male} / \mathrm{female}$ samples may contain approximately $90 \% / 95 \%$ analytical completeness, respectively, of a group urinary proteome.

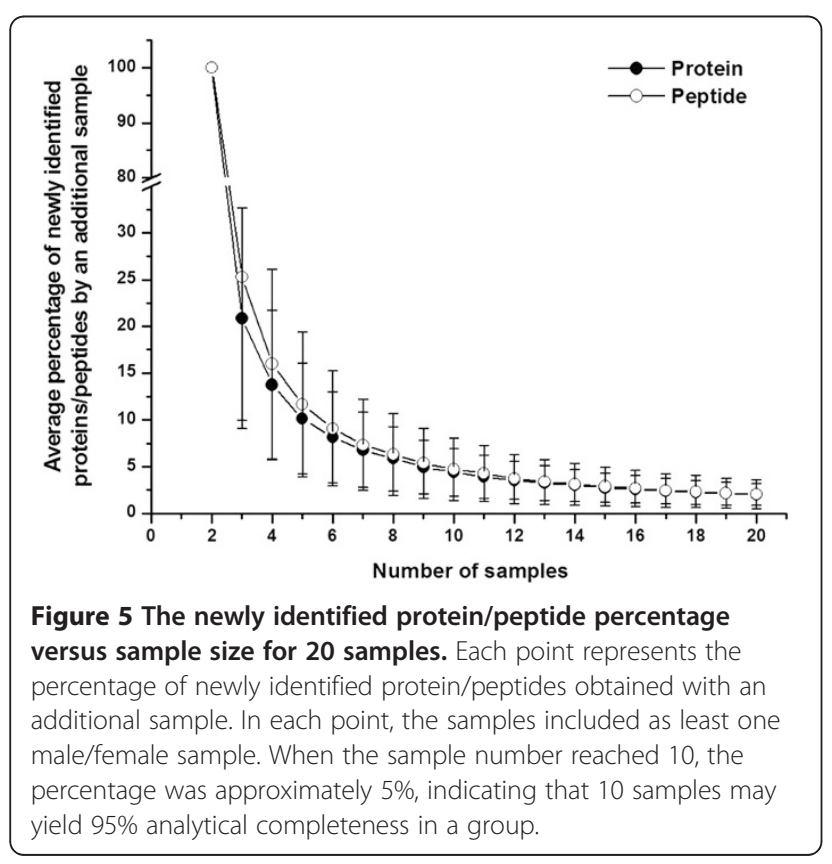

To define the inter-individual and inter-gender variation more comprehensively, further qualitative analysis was performed. Figure 6A shows the pairwise protein overlap rate of the 21 samples. For visualization, the rates were color-coded. The higher overlap rates were clearly in the intra-gender region, and the pooled male samples had higher overlap rates with male samples. Hierarchical clustering analysis based on the same qualitative data showed that the male/female samples clustered together (Figure 6B). These results indicate that the male and female urinary proteome may have different patterns.

\section{The minimal sample number for a group by quantitative analysis}

Quantitative analysis has been widely used in clinical urinary proteomic studies. In these studies, hypothesis test (usually $t$ test) is commonly used to identify whether a protein expressed significantly different between the disease and control group. Two types of error occur in a hypotheses test: the false positive error $\alpha$ and the false negative error $\beta . \alpha$ is also called the significance level, whereas $1-\beta$ refers to the power of the statistical test. Controlling both of these errors is crucial to the success of a proteomic study. To achieve this goal, enough number of samples must be included in the study. The minimal sample size in each group can be calculated based on the level of variation between samples, the expected fold change, the significance level and the statistical power [25].

In proteomic studies, typically hundreds or thousands of proteins/peaks are needed for the hypothesis test of their expression level. The chance of the false positive results increases significantly when multiple hypothesis tests are needed to be performed. Therefore false 


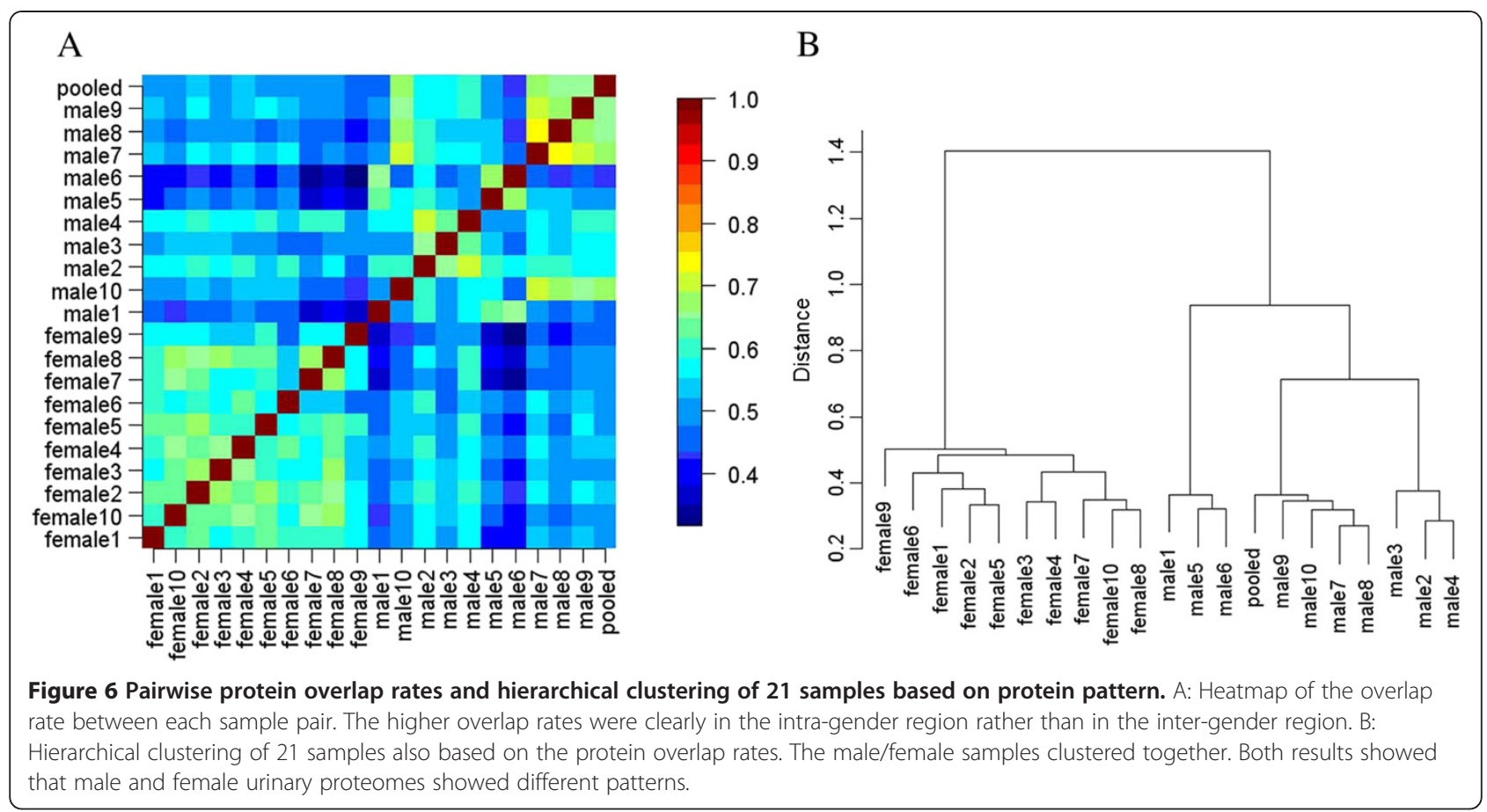

discovery rate (FDR) of the entire quantitative proteomic analysis should be calculated and controlled to an acceptable level [28].

In this report a semi-quantitative method (spectrum count, SC) was used to calculate the coefficient of variation $(\mathrm{CV})$ between samples. Because $\mathrm{SC}$ was relatively accurate for evaluating highly abundant proteins, only proteins identified in more than $80 \%$ of the 291 LC-MS/ MS runs were included, which was a total of 31 proteins. The median CV of these proteins was then used to calculate the minimal sample number. For male, female, and normal groups, the median CV was $66.2 \%, 58.2 \%$, and $70.6 \%$, respectively. The minimal sample number at a 5\%FDR is shown in Table 2. When the fold change was 2 , the average minimal sample number was 16,13 , and 18 for the male, female, and normal groups, respectively. However, when the fold change was 1.5 , the average minimal sample number increased to 58,46 , and 66 for these groups, respectively.

To estimate the minimal sample number for proteins with lower abundance, western blot was used to measure three proteins (alpha 1 antitrypsin, ceruloplasmin, and

Table 2 The estimated minimal sample number per group for quantitative analysis based on spectrum count method, where power is the power of statistical test, $a$ is the significance level, and $\pi$ refers to the estimated proportion of truly differentially expressed proteins among all of the identified proteins

\begin{tabular}{|c|c|c|c|c|c|c|c|}
\hline fold change & power & $a$ & $\pi$ & FDR & normal group & male group & female group \\
\hline \multirow[t]{6}{*}{2} & 0.8 & 0.001 & 0.05 & $2.31 \%$ & 20 & 18 & 15 \\
\hline & 0.8 & 0.005 & 0.1 & $5.33 \%$ & 16 & 14 & 12 \\
\hline & 0.8 & 0.01 & 0.2 & $4.76 \%$ & 14 & 12 & 10 \\
\hline & 0.9 & 0.001 & 0.05 & $2.06 \%$ & 24 & 22 & 17 \\
\hline & 0.9 & 0.005 & 0.1 & $4.76 \%$ & 19 & 17 & 14 \\
\hline & 0.9 & 0.01 & 0.2 & $4.26 \%$ & 17 & 15 & 12 \\
\hline \multirow[t]{6}{*}{1.5} & 0.8 & 0.001 & 0.05 & $2.31 \%$ & 71 & 63 & 49 \\
\hline & 0.8 & 0.005 & 0.1 & $5.33 \%$ & 56 & 49 & 39 \\
\hline & 0.8 & 0.01 & 0.2 & $4.76 \%$ & 49 & 43 & 34 \\
\hline & 0.9 & 0.001 & 0.05 & $2.06 \%$ & 87 & 77 & 60 \\
\hline & 0.9 & 0.005 & 0.1 & $4.76 \%$ & 69 & 61 & 48 \\
\hline & 0.9 & 0.01 & 0.2 & $4.26 \%$ & 62 & 54 & 43 \\
\hline
\end{tabular}


beta-2-microglobulin). These three proteins were identified in less than $40 \%$ of the LC-MS/MS runs and the CVs of SC were $170.6 \%, 279.1 \%$, and $222 \%$, respectively. However, based on the western blot analysis (Figure 7), the CVs were $81.2 \%, 101.1 \%$, and $93 \%$, respectively, indicating that quantitative western blot was more accurate than SC for proteins with low abundance. The average minimal sample number for these three proteins was 30 with a 2 -fold change and 110 with a 1.5 -fold change, indicating that more samples were necessary to obtain significant results for proteins of low abundance than those of high abundance (Table 3).

\section{Discussion}

In recent years, clinical urinary proteomic analyses have been widely used to discover biomarkers. A thorough and representative urinary proteome database of normal human samples is critically important as the background of a disease proteome for discovery proteomics and the source of candidate proteins/peptides for targeted proteomics. Since 2001, a number of groups have addressed this issue, and more than 2500 proteins have been identified from the normal human urinary proteome. However, there are still some important aspects that need to be defined.

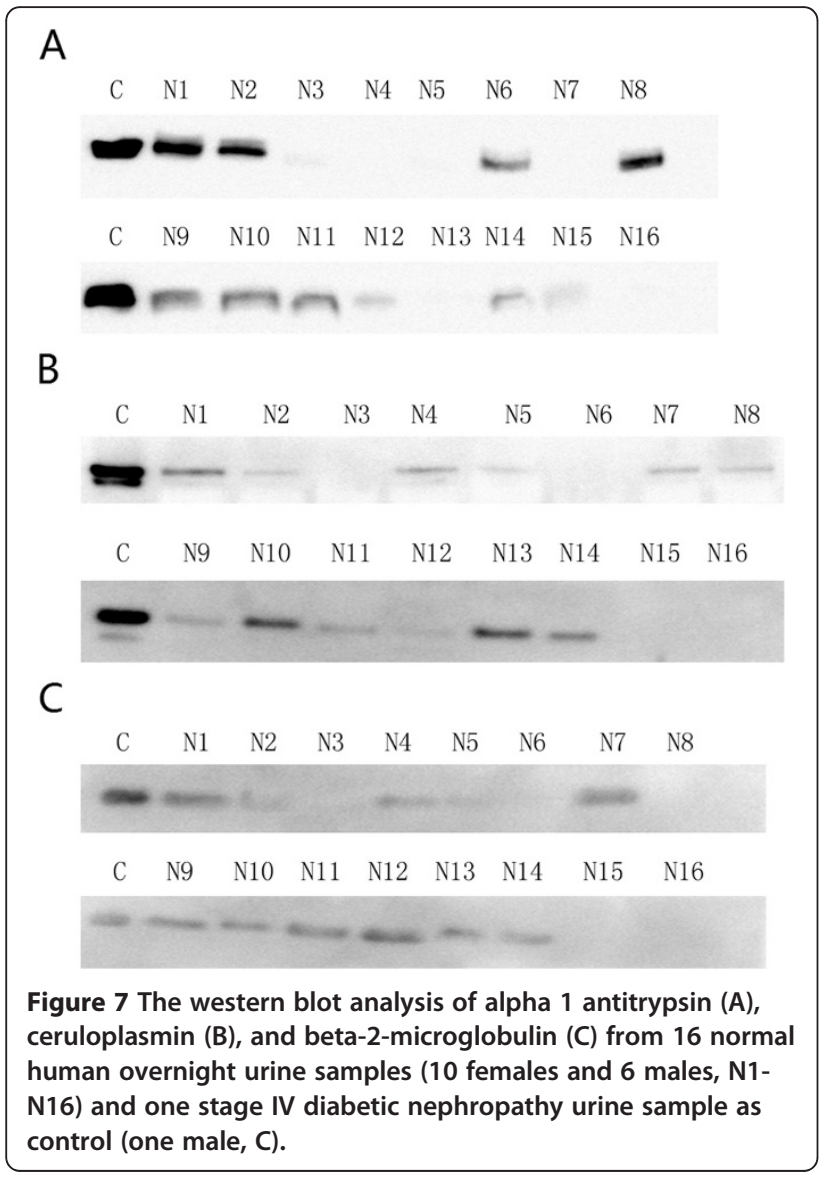

To construct a representative urinary proteome, it is necessary to define the minimal sample number. Too few samples may present individual-specific proteins that do not represent the group pattern. Previous studies [15-17] have used various sample numbers ranging from one to over ten. However, because this issue has not been thoroughly assessed, the minimal sample number was unknown. In this study, inter-individual and intergender variations were taken into consideration for qualitative analysis to achieve a representative urinary proteome. We used replicate LC/MS/MS analyses of 20 urine samples from healthy volunteers to define the minimal sample number needed. The results showed that 9 male/female samples may contain approximately $95 \%$ analytical completeness of a male/female group. For a group, 10 samples can achieve 95\% analytical completeness. Importantly, the results of this study may be helpful for constructing a new urinary proteome database or evaluating an existing database. The universal application of these conclusions should be cautioned for several reasons. First, technical variations factors, including sample preparation, LC separation, mass spectrometer detection, and data processing can affect the final identification results. The conclusions of this study are based on the results obtained with 1DLC separation and a low sensitivity and resolution mass spectrometer (LTQ $\mathrm{XL}$ ). Any change in these factors, such as using an instrument with high sensitivity and high resolution (i.e. Orbitrap or TripleTOF 5600), might result in a different conclusion. For example, in this report a total of 867 proteins were identified with one-dimensional separation (1DLC) and low resolution instrument (LTQ XL). Kentsis et al. [29] identified 2362 proteins using three-dimensional separation (centrifugation, SDS-PAGE, and 1DLC) and a high resolution instrument (LTQ Orbitrap XL). Thus, with more separation approaches and a more accurate instrument, a substantially greater number of proteins could be identified and more useful information might be obtained. On the other hand, it is well known that the urinary proteome had great biological variation. In this study, only interindividual and inter-gender variations were taken into consideration, and other biological variations (such as age, hormone level, exercise, and others [30]) may also have a marked impact on the results and increase the sample number. Therefore, the conclusion presented here represents a preliminary result that may be the minimal sample number needed. If other variation factors are included, the minimal sample number may increase.

Another important issue regarding a normal urinary proteome database is the quantitative information. For clinical research, the aim is generally focused on identifying disease-related biomarkers. The quantitative information of each protein would be helpful to define biological and technical variations so that the differential 
Table 3 The estimated minimal sample number per group for five proteins based on western blot, where power is the power of statistical test, $a$ is the significance level, and $\pi$ refers to the estimated proportion of truly differentially expressed proteins among all of the identified proteins.

\begin{tabular}{|c|c|c|c|c|c|c|c|}
\hline \multirow[t]{2}{*}{ fold change } & \multirow[t]{2}{*}{ power } & \multirow[t]{2}{*}{$a$} & \multirow[t]{2}{*}{$\pi$} & \multirow[t]{2}{*}{ FDR } & \multirow{2}{*}{$\frac{\text { alpha } 1}{\text { antitrypsin }}$} & \multirow[t]{2}{*}{ ceruloplasmin } & \multirow{2}{*}{$\frac{\text { beta-2 }}{\text { microglobulir }}$} \\
\hline & & & & & & & \\
\hline \multirow[t]{6}{*}{2} & 0.8 & 0.001 & 0.05 & $2.31 \%$ & 26 & 38 & 33 \\
\hline & 0.8 & 0.005 & 0.1 & $5.33 \%$ & 20 & 30 & 26 \\
\hline & 0.8 & 0.01 & 0.2 & $4.76 \%$ & 18 & 26 & 22 \\
\hline & 0.9 & 0.001 & 0.05 & $2.06 \%$ & 31 & 46 & 39 \\
\hline & 0.9 & 0.005 & 0.1 & $4.76 \%$ & 25 & 37 & 31 \\
\hline & 0.9 & 0.01 & 0.2 & $4.26 \%$ & 22 & 33 & 28 \\
\hline \multirow[t]{6}{*}{1.5} & 0.8 & 0.001 & 0.05 & $2.31 \%$ & 93 & 143 & 121 \\
\hline & 0.8 & 0.005 & 0.1 & $5.33 \%$ & 73 & 111 & 95 \\
\hline & 0.8 & 0.01 & 0.2 & $4.76 \%$ & 64 & 98 & 83 \\
\hline & 0.9 & 0.001 & 0.05 & $2.06 \%$ & 113 & 174 & 148 \\
\hline & 0.9 & 0.005 & 0.1 & $4.76 \%$ & 91 & 139 & 118 \\
\hline & 0.9 & 0.01 & 0.2 & $4.26 \%$ & 81 & 124 & 105 \\
\hline
\end{tabular}

proteins with statistical significance in a group could be identified. In addition, the false differential proteins found due to the high variation in the group could be excluded. To date, most normal urinary proteome analyses have been qualitative studies, and only the study by Nagaraj et al. [15] provided overall quantitative information of each protein using a peak intensity method. In this report, we used SC and western blot to assess quantitative information of high and low abundance proteins, respectively, and to estimate the minimal sample number needed for quantitative analysis. For high abundance proteins, the average minimal sample number was 18 with a 2 -fold change, and for the proteins of low abundance, the number was 30 with a 2-fold change. These results indicated that a higher minimal sample number is required to obtain statistical significance when detecting proteins of low abundance. We also attempted to estimate the minimal sample number using the Nagaraj et al. [15] data. With 66\% inter-individual CV, the minimal average sample number was 16 with a 2-fold change and 58 with a 1.5-fold change, among all the acceptable levels of FDR and statistical power. These results were similar to our results for proteins of high abundance using the SC method. However, the sample number, separation method, MS instrument, data processing software and protein number used for quantitative analysis were different between these two studies. Considering that there were other quantitative methods, such as iTRAQ and TMT, it is difficult to conclude that the minimal sample number for urinary proteome quantitative analysis. Therefore, it is necessary to evaluate the variations of various quantitative methods in the future to define a proper minimal sample number for clinical research.
Our previous work [4] showed that it was hard to define the difference between the male and female urinary proteomes, except for the identification of several malespecific proteins. In addition, recent studies by both LC/ MS/MS [5] and 2DE [6] approaches also failed to identify these differences. In this study, the protein overlap rates among the 21 samples and the result of hierarchical clustering analysis allowed us to separate male and female samples into two groups, indicating a difference between the male and female urinary proteome pattern. However, because this study was only based on 1DLC/ MS/MS analysis and low-resolution mass spectrometry, the conclusion should be confirmed with additional experiments before being universally applied. In addition, considering the existence of male-specific proteins, it is important that the ratio of male and female samples is balanced when constructing a database.

The choice of pooled or individual sample was also an important issue. Since the proteome is known to have substantial biological variation, an appropriate number of samples should be analyzed for proteomic analysis. However, a few years ago the throughput of proteomic techniques was limited, and in order to circumvent this problem, samples were pooled [31]. Previous reports on cell lines [32] or tissues [33,34] by 2DE showed that pooling could reduce biological variation. On the other hand, Diz et al. [34] as well as our previous study [4] showed that pooling samples may lead to a loss of information through sample dilution. In this study, the pooled male sample was found to be clustered with male samples and closest to the samples from Male 7-10, indicating that a pooled sample may not adequately represent the pattern of all individual samples. Therefore, the results from a pooled sample should be carefully 
assessed before being applied to other experiments. In recent years, with the application of instruments having high sensitivity and high resolution (such as Orbitrap), high-throughput urinary proteome analysis has become possible. Nagaraj et al. [5] identified over 800 proteins in a 4h 1DLC/MS/MS analysis using an LTQ Orbitrap XL. Therefore, the use of individual samples is recommended in future work.

\section{Conclusion}

With the wide application of the urinary proteome in clinical research, the construction of a representative and informative normal urinary proteome database has become critically important. Considering the interindividual and inter-gender variation of the urinary proteome, we used replicate 1DLC/MS/MS to analyze 10 male and 10 female samples for qualitative analysis, and found that in order to achieve a representative urinary database the minimal sample number was estimated to be 10. In addition, the number of male and female samples in a group had better be balanced. For quantitative analysis, proteins of low abundance showed greater variation and required more samples to obtain statistical significance than proteins of high abundance. In addition, different quantitative analyses exhibited different technical variation, and therefore the minimal sample number should be evaluated in conjunction with the quantitative method being used. With the need of quantitative information for each protein in a group and the application of high sensitivity and resolution mass spectrometry, the high-throughput analysis of individual urinary proteomes using different quantitative methods would greatly benefit future clinical urinary proteome studies.

\section{Additional files}

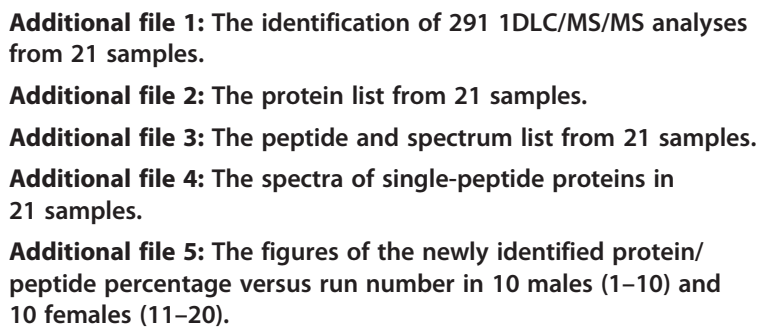

\section{Authors' contributions}

Xuejiao Liu participated in sample collection, sample preparations, data collection, and drafting of the manuscript. Chen Shao was involved in data collection, data analysis, and the drafting of the manuscript. Lilong Wei participated in sample preparations and data collection. Jindan Duan and Shuzhen Wu were involved in sample collection and sample preparations. Xuewang Li participated in the design of the experiments. Wei Sun and
Mingxi Li participated in the design of the experiments and the drafting of the manuscript. All the authors approved the order of the authorship. All authors read and approved the final manuscript.

\section{Acknowledgements}

This work was supported by grants from National Natural Science Foundation (No. 30970650 and 31200614), a Foundation for the Author of National Excellent Doctoral Dissertation of P.R. China (No. 2007B64).

\section{Author details}

${ }^{1}$ Department of Nephrology, Peking Union Medical College Hospital, Chinese Academy of Medical Sciences, No. 1 Shuaifuyan, Wangfujing Street, Beijing, China. ${ }^{2}$ Department of Physiology and Pathophysiology, National Key Laboratory of Medical Molecular Biology, Institute of Basic Medical Sciences Chinese Academy of Medical Sciences/School of Basic Medicine Peking Union Medical College, 5 Dong Dan San Tiao, Beijing, China. ${ }^{3}$ Clinical laboratory, China-Japan Friendship Hospital, 2 Yinghua Dongjie, Hepingli, Beijing, China. ${ }^{4}$ Core facility of instrument, Institute of Basic Medical Sciences Chinese Academy of Medical Sciences/School of Basic Medicine Peking Union Medical College, 5 Dong Dan San Tiao, Beijing, China.

Received: 30 June 2012 Accepted: 24 October 2012

Published: 21 November 2012

\section{References}

1. Oh J, Pyo JH, Jo EH, Hwang SI, Kang SC, Jung JH, Park EK, Kim SY, Choi JY, Lim J: Establishment of a near-standard two-dimensional human urine proteomic map. Proteomics 2004, 4(11):3485-3497. Nov.

2. Thongboonkerd V, Chutipongtanate S, Kanlaya R: Systematic evaluation of sample preparation methods for gel-based human urinary proteomics: quantity, quality, and variability. J Proteome Res 2006, 5(1):183-191. Jan.

3. Khan A, Packer $\mathrm{NH}$ : Simple urinary sample preparation for proteomic analysis. J Proteome Res 2006, 5(10):2824-2838. Oct.

4. Sun W, Chen Y, Li F, Zhang L, Yang R, Zhang Z, Zheng D, Gao Y: Dynamic urinary proteomic analysis reveals stable proteins to be potential biomarkers. Proteomics Clin Appl 2009, 3(3):370-382. Mar.

5. Nagaraj N, Mann M: Quantitative analysis of the intra- and interindividual variability of the normal urinary proteome. J Proteome Res 2011, 10(2):637-645. Feb 4.

6. Molina L, Salvetat N, Ameur RB, Peres S, Sommerer N, Jarraya F, Ayadi H, Molina F, Granier C: Analysis of the variability of human normal urine by 2D-GE reveals a "public" and a "private" proteome. J Proteomics 2011, 75(1):70-80. Dec 10.

7. Spahr CS, Davis MT, McGinley MD, Robinson JH, Bures EJ, Beierle J, Mort J, Courchesne PL, Chen K, Wahl RC, Yu W, Luethy R, Patterson SD: Towards defining the urinary proteome using liquid chromatography-tandem mass spectrometry. I. Profiling an unfractionated tryptic digest. Proteomics 2001, 1(1):93-107. Jan.

8. O'Riordan E, Orlova TN, Podust VN, Chander PN, Yanagi S, Nakazato M, Hu R, Butt K, Delaney V, Goligorsky MS: Characterization of urinary peptide biomarkers of acute rejection in renal allografts. Am J Transplant 2007, 7(4):930-940. Apr.

9. Rossing K, Mischak H, Dakna M, Zürbig P, Novak J, Julian BA, Good DM, Coon JJ, Tarnow L, Rossing P: PREDICTIONS Network. Urinary proteomics in diabetes and CKD. J Am Soc Nephrol 2008, 19(7):1283-1290.

10. Decramer S, Wittke S, Mischak H, Zürbig P, Walden M, Bouissou F, Bascands $J$, Schanstra JP: Predicting the clinical outcome of congenital unilateral ureteropelvic junction obstruction in newborn by urinary proteome analysis. Nat Med 2006, 12(4):398-400. Apr.

11. Irmak S, Tilki D, Heukeshoven J, Oliveira-Ferrer L, Friedrich $M$, Huland $H$, Ergün S: Stage-dependent increase of orosomucoid and zinc-alpha2glycoprotein in urinary bladder cancer. Proteomics 2005, 5(16):4296-4304. Nov.

12. Theodorescu D, Schiffer E, Bauer HW, Douwes F, Eichhorn F, Polley R, Schmidt T, Schöfer W, Zürbig P, Good DM, Coon JJ, Mischak H: Discovery and validation of urinary biomarkers for prostate cancer. Proteomics Clin App/ 2008, 2(4):556-570. Mar 7.

13. Kaiser T, Kamal H, Rank A, Kolb HJ, Holler E, Ganser A, Hertenstein B, Mischak H, Weissinger EM: Proteomics applied to the clinical follow-up of patients after allogeneic hematopoietic stem cell transplantation. Blood 2004, 104(2):340-349. Jul 15. 
14. Zimmerli LU, Schiffer E, Zürbig P, Good DM, Kellmann M, Mouls L, Pitt AR, Coon JJ, Schmieder RE, Peter KH, Mischak H, Kolch W, Delles C, Dominiczak AF: Urinary proteomic biomarkers in coronary artery disease. Mol Cell Proteomics 2008, 7(2):290-298. Feb.

15. Marimuthu A, O'Meally RN, Chaerkady R, Subbannayya Y, Nanjappa V, Kumar P, Kelkar DS, Pinto SM, Sharma R, Renuse S, Goel R, Christopher R, Delanghe B, Cole RN, Harsha HC, Pandey A: A comprehensive map of the human urinary proteome. J Proteome Res 2011, 10(6):2734-2743. Jun 3.

16. Adachi J, Kumar C, Zhang Y, Olsen JV, Mann M: The human urinary proteome contains more than 1500 proteins, including a large proportion of membrane proteins. Genome Biol 2006, 7(9):R80.

17. Gonzales PA, Pisitkun T, Hoffert JD, Tchapyjnikov D, Star RA, Kleta R, Wang NS, Knepper MA: Large-scale proteomics and phosphoproteomics of urinary exosomes. J Am Soc Nephrol 2009, 20(2):363-379. Feb.

18. Liu H, Sadygov RG, Yates JR 3rd: A model for random sampling and estimation of relative protein abundance in shotgun proteomics. Anal Chem 2004, 76(14):4193-4201. Jul 15.

19. Brunner E, Ahrens $\mathrm{CH}$, Mohanty S, Baetschmann H, Loevenich S, Potthast F, Deutsch EW, Panse C, de Lichtenberg U, Rinner O, Lee H, Pedrioli PG, Malmstrom J, Koehler K, Schrimpf S, Krijgsveld J, Kregenow F, Heck AJ, Hafen E, Schlapbach R, Aebersold R: A high-quality catalog of the Drosophila melanogaster proteome. Nat Biotechnol 2007, 25(5):576-583. May.

20. Durr E, Yu J, Krasinska KM, Carver LA, Yates JR, Testa JE, Oh P, Schnitzer JE: Direct proteomic mapping of the lung microvascular endothelial cell surface in vivo and in cell culture. Nat Biotechnol 2004, 22(8):985-992. Aug.

21. Sun W, Gao S, Wang L, Chen Y, Wu S, Wang X, Zheng D, Gao Y: Microwave-assisted protein preparation and enzymatic digestion in proteomics. Mol Cell Proteomics 2006, 5(4):769-776. Apr.

22. Eng JK, McCormak AM, Yates JR III: An approach to correlate tandem mass spectral data of peptides with amino acid sequences in a protein database. J Am Soc Mass Spectrom 1994, 5:976-989.

23. Kersey PJ, Duarte J, Williams A, Karavidopoulou Y, Birney E, Apweiler R: The international protein index: an integrated database for proteomics experiments. Proteomics 2004, 4(7):1985-1988. Jul.

24. Nesvizhskii Al, Aebersold R: Interpretation of shotgun proteomic data: the protein inference problem. Mol Cell Proteomics 2005, 4(10):1419-1440. Oct.

25. Anon: Statistical power analysis for the behavioral-sciences - Cohen. J Percept Mot Skills 1988, 67:1007.

26. Elias JE, Gibbons FD, King OD, Roth FP, Gygi SP: Intensity-based protein identification by machine learning from a library of tandem mass spectra. Nat Biotechnol 2004, 22:214-219.

27. Sun W, Li F, Wu S, Wang X, Zheng D, Wang J, Gao Y: Human urine proteome analysis by three separation approaches. Proteomics 2005, 5(18):4994-5001. Dec.

28. Cairns DA, Barrett JH, Billingham LJ, Stanley AJ, Xinarianos G, Field JK, Johnson PJ, Selby PJ, Banks RE: Sample size determination in clinical proteomic profiling experiments using mass spectrometry for class comparison. Proteomics 2009, 9(1):74-86. Jan.

29. Kentsis A, Monigatti F, Dorff K, Campagne F, Bachur R, Steen H: Urine proteomics for profiling of human disease using high accuracy mass spectrometry. Proteomics Clin Appl 2009, 3(9):1052-1061. Sep 1.

30. Kentsis A: Challenges and opportunities for discovery of disease biomarkers using urine proteomics. Pediatr Int 2011, 53(1):1-6. Feb.

31. Zolg W: The proteomic search for diagnostic biomarkers: lost in translation? Mol Cell Proteomics 2006, 5(10):1720-1726. Oct.

32. Weinkauf M, Hiddemann W, Dreyling M: Sample pooling in 2-D gel electrophoresis: a new approach to reduce nonspecific expression background. Electrophoresis 2006, 27(22):4555-4558. Nov.

33. Karp NA, Lilley KS: Investigating sample pooling strategies for DIGE experiments to address biological variability. Proteomics 2009, 9(2):388-397. Jan.

34. Diz AP, Truebano M, Skibinski DO: The consequences of sample pooling in proteomics: an empirical study. Electrophoresis 2009, 30(17):2967-2975. Sep.

doi:10.1186/1477-5956-10-70

Cite this article as: Liu et al.: An individual urinary proteome analysis in normal human beings to define the minimal sample number to represent the normal urinary proteome. Proteome Science 2012 10:70.

\section{Submit your next manuscript to BioMed Central and take full advantage of:}

- Convenient online submission

- Thorough peer review

- No space constraints or color figure charges

- Immediate publication on acceptance

- Inclusion in PubMed, CAS, Scopus and Google Scholar

- Research which is freely available for redistribution 